\title{
Association of Amyloid Pathology With Myelin Alteration in Preclinical Alzheimer Disease
}

Douglas C. Dean III, PhD; Samuel A. Hurley, PhD; Steven R. Kecskemeti, PhD; J. Patrick O'Grady; Cristybelle Canda; Nancy J. Davenport-Sis; Cynthia M. Carlsson, MD, MS; Henrik Zetterberg, MD, PhD; Kaj Blennow, MD, PhD; Sanjay Asthana, MD; Mark A. Sager, MD; Sterling C. Johnson, PhD; Andrew L. Alexander, PhD;

Barbara B. Bendlin, PhD

IMPORTANCE The accumulation of aggregated $\beta$-amyloid and tau proteins into plaques and tangles is a central feature of Alzheimer disease (AD). While plaque and tangle accumulation likely contributes to neuron and synapse loss, disease-related changes to oligodendrocytes and myelin are also suspected of playing a role in development of AD dementia. Still, to our knowledge, little is known about AD-related myelin changes, and even when present, they are often regarded as secondary to concomitant arteriosclerosis or related to aging.

OBJECTIVE To assess associations between hallmark AD pathology and novel quantitative neuroimaging markers while being sensitive to white matter myelin content.

DESIGN, SETTING, AND PARTICIPANTS Magnetic resonance imaging was performed at an academic research neuroimaging center on a cohort of 71 cognitively asymptomatic adults enriched for AD risk. Lumbar punctures were performed and assayed for cerebrospinal fluid (CSF) biomarkers of $A D$ pathology, including $\beta$-amyloid 42 total tau protein, phosphorylated tau 181, and soluble amyloid precursor protein. We measured whole-brain longitudinal and transverse relaxation rates as well as the myelin water fraction from each of these individuals.

MAIN OUTCOMES AND MEASURES Automated brain mapping algorithms and statistical models were used to evaluate the relationships between age, CSF biomarkers of AD pathology, and quantitative magnetic resonance imaging relaxometry measures, including the longitudinal and transverse relaxation rates and the myelin water fraction.

RESULTS The mean (SD) age for the 19 male participants and 52 female participants in the study was 61.6 (6.4) years. Widespread age-related changes to myelin were observed across the brain, particularly in late myelinating brain regions such as frontal white matter and the genu of the corpus callosum. Quantitative relaxometry measures were negatively associated with levels of CSF biomarkers across brain white matter and in areas preferentially affected in AD. Furthermore, significant age-by-biomarker interactions were observed between myelin water fraction and phosphorylated tau $181 / \beta$-amyloid 42 , suggesting that phosphorylated tau $181 / \beta$-amyloid 42 levels modulate age-related changes in myelin water fraction.

CONCLUSIONS AND RELEVANCE These findings suggest amyloid pathologies significantly influence white matter and that these abnormalities may signify an early feature of the disease process. We expect that clarifying the nature of myelin damage in preclinical AD may be informative on the disease's course and lead to new markers of efficacy for prevention and treatment trials.
JAMA Neurol. 2017;74(1):41-49. doi:10.1001/jamaneurol.2016.3232

Published online November 14, 2016 $\leftarrow$

Editorial page 17

Supplemental content
Author Affiliations: Author affiliations are listed at the end of this article.

Corresponding Author: Douglas C. Dean III, PhD, Waisman Center, University of Wisconsin-Madison, Madison, WI 53705 (deaniii@wisc .edu). 
$\mathrm{T}$ he progression of Alzheimer disease $(\mathrm{AD})$ pathology occurs several years before the development of dementia. According to the amyloid-cascade hypothesis, the disruption of critical metabolic processes that lead to the ultimate neurodegeneration in $\mathrm{AD}$ is initiated by the accumulation of aggregated $\beta$-amyloid 1-42 (A $\beta 1-42)$ and the assembly of neurofibrillary tangles. ${ }^{1}$ Such processes are clearly detrimental to neuronal cell bodies, dendrites, and axonal processes; however, myelin and myelin-producing oligodendrocytes may be equally vulnerable to the impairments caused by $\mathrm{A} \beta$ and tau protein hyperphosphorylation. ${ }^{2}$ White matter hyperintensities (WMHs) play a role in symptom presentation but are not core features of AD pathology. ${ }^{3}$ Postmortem and in vivo magnetic resonance imaging (MRI) studies have substantiated recent hypotheses of white matter involvement in $\mathrm{AD}$, finding reduced white matter volume and alterations of white matter microstructure. ${ }^{4-6}$ Still, little is known about the relationship between $A \beta$ pathology and myelin alteration.

Recent advancements of cerebrospinal fluid (CSF) biomarkers show promise for early detection of $\mathrm{AD}$ pathology. Concentrations of specific proteins within CSF, such as $\mathrm{A} \beta 42$, total tau protein (Ttau), and phosphorylated tau 181 (Ptau181), are related to the core pathology of $\mathrm{AD}^{7-9}$ and differentiate patients with AD from healthy, age-matched controls. ${ }^{10}$ Cerebrospinal fluid biomarkers have additionally been linked to measures obtained with volumetric MRI and diffusion tensor imaging, suggesting that CSF biomarkers are sensitive to structural brain changes during the preclinical stages, onset, and progression of $\mathrm{AD} .{ }^{4}$ Relationships between CSF biomarkers and brain structure are increasingly important ${ }^{4,11}$; however, while MRI techniques provide detailed anatomical and microstructural insight, they are influenced by a broad range of microstructural changes. ${ }^{12}$ Thus, the biological interpretation of such associations is challenging.

The breakdown of the myelin sheath may be an early phenomenon in $\mathrm{AD},{ }^{5,13}$ but more clinical studies are needed, especially in the preclinical stage. Recent quantitative MRI measures, such as longitudinal and transverse relaxation rates $\left(R_{1}\right.$ and $\mathrm{R}_{2}$, respectively) and the myelin water fraction (MWF), may provide increased sensitivity to myelin content, ${ }^{14,15}$ and these may offer new insights regarding the role of myelin vulnerability in the pathogenesis of AD. Examining relationships between white matter measures and CSF biomarkers related to AD pathology-such as A $\beta 42$ (reflecting cortical amyloid deposition), Ttau (as a marker for the intensity of neurodegeneration), and Ptau181 (correlating with tangle burden) ${ }^{16}$-could provide an appreciation for the extent and timing of myelinated white matter damage in $\mathrm{AD}$. To our knowledge, no studies to date have explored such relations at the preclinical stage of AD. However, evidence of such relationships may support and transform the current understanding of the involvement of myelinated white matter and its alterations during the development of $\mathrm{AD}$.

We report results from 71 late-middle-aged asymptomatic adults who underwent CSF collection via lumbar puncture $^{17}$ and novel MRI using the Multicomponent Driven Equilibrium Single Pulse Observation of $\mathrm{T}_{1}$ and $\mathrm{T}_{2}$

\section{Key Points}

Question What are the underlying relationships between white matter microstructure and, more specifically, myelin content and cerebrospinal fluid biomarkers of Alzheimer disease?

Findings In this cohort study of asymptomatic individuals with genetic risk factors for Alzheimer disease, significant associations were observed between quantitative neuroimaging measures, including the myelin water fraction and cerebrospinal fluid biomarkers of Alzheimer pathology.

Meaning Mechanisms of amyloid pathology influence and alter the white matter microstructure, which may indicate an early feature of Alzheimer disease.

(mcDESPOT) technique. These data were acquired to examine AD pathology as indexed by CSF biomarkers and the MWF, a surrogate measure of myelin content. ${ }^{15,18}$ Guided by prior models and observations, ${ }^{4}$ we hypothesized that the deposition of $A \beta 42$ and the hyperphosphorylation of tau protein would affect oligodendrocytes and alter myelin sheath integrity. Hence, we predicted decreased $\mathrm{A} \beta 42$ and elevated Ttau and $\mathrm{Ptau}_{181}$ levels would be associated with decreased MWF. We additionally hypothesized that proteins that precede A $\beta 42$ formation, including elevated cleavage of the amyloid precursor protein by $\beta$-secretase that results in higher soluble amyloid precursor protein (sAPP $\beta$ ), may stimulate processes of white matter alteration and be associated with myelin content alterations in the preclinical phase.

\section{Methods}

A total of 147 participants were enrolled in the study, from which 71 asymptomatic participants (19 males) between the ages of 48 and 72 years (mean [SD] age $=61.6$ [6.4] years) were included based on the availability of assayed CSF and mcDESPOT imaging. Healthy community volunteers were recruited from the Wisconsin Alzheimer Disease Research Center and the Wisconsin Registry for Alzheimer's Prevention study ${ }^{17}$ Participants underwent assessments that included neuropsychological testing, apolipoprotein E (APOE) genotyping, laboratory tests, clinical measurements, and comprehensive health history characterization. ${ }^{17}$ Twenty-eight participants (39\%) were carriers of at least 1 APOE $\varepsilon 4$ allele and 54 participants $(76 \%)$ had a parental history of AD. Inclusion criteria consisted of the following: a prior visit for lumbar puncture, no contraindications for MRI, and a subsequent normal MRI scan finding. Cardiovascular health was assessed using the Framingham cardiovascular risk score and further inclusion was limited to participants who scored 27 or greater on the Mini-Mental State Examination. ${ }^{19}$ Written informed consent was obtained from all study participants and the study procedures were performed under guidelines approved by the University of Wisconsin Health Sciences institutional review board. Additional participant information is available in the eMethods in the Supplement while demographic information is provided in the Table. 


\section{CSF Collection}

Cerebrospinal fluid was collected via lumbar puncture using a Sprotte 25- or 24-gauge spinal needle at the L3/4 or L4/5 level of the spinal column the morning after a 12-hour overnight fast. Lumbar punctures were performed an average of 5.86 days prior to brain imaging. Approximately $22 \mathrm{~mL}$ of CSF were extracted, mixed, and centrifuged at $2000 \mathrm{~g}$ for 10 minutes. Supernatants were frozen in polypropylene tubes in $0.5-\mathrm{mL}$ aliquots and stored at $-80^{\circ} \mathrm{C}$. Samples were aliquoted in sterile polypropylene collection tubes and stored in a 280uC freezer. The samples were subsequently sent in a single batch to the Sahlgrenska University Hospital at the University of Gothenburg in Sweden, where CSF was assayed for A $\beta 42$, Ttau, and Ptau181 using commercially available enzyme-linked immunosorbent assay methods (INNOTEST assays; Fujiurebio) ${ }^{20}$ Cerebrospinal fluid SAPP- $\beta$ was measured using the Meso Scale Discovery Multiplex Soluble APP assay according to the manufacturer's instructions. Board-certified laboratory technicians, blinded to clinical information, analyzed all samples according to protocols approved by the Swedish Board of Accreditation and Conformity Assessment. One batch of reagents was used yielding intra-assay coefficients of less than $10 \%$ variation.

\section{MRI Data Acquisition and Processing}

Participants were imaged on a 3 T General Electric MR750 Discovery scanner (General Electric Healthcare) with an 8-channel receive-only head coil (Nova Medical). The mcDESPOT protocol included both spoiled gradient-recalled echo images and balanced steady-state free precession images acquired over multiple flip angles (eFigure 1 in the Supplement). ${ }^{15}$ All images were acquired sagittally and shared a common field of view of $25.6 \mathrm{~cm}$ by $25.6 \mathrm{~cm}$ by $16.8 \mathrm{~cm}$ with an isotropic voxel resolution of $2 \mathrm{~mm}^{3}$. Acquisition time for the mcDESPOT protocol was approximately 8 minutes per participant. Additional sequence-specific parameters are provided in the eMethods in the Supplement.

$R_{1}, R_{2}$, and MWF maps were calculated and aligned to the Montreal Neurological Institute template space, as described in eMethods in the Supplement. Total WMH volume was also measured using high-resolution T1-weighted and T2weighted images and the Lesion Segmentation Toolbox version 1.2.2 in Statistical Parametric Mapping 8 (http://www.fil .ion.ucl.ac.uk/spm/), normalized by the total intracranial volume and log-transformed for the inclusion of statistical analyses.

\section{Statistical Analysis}

Relationships of Age, CSF Biomarkers, and Imaging Measures

The single greatest risk factor for AD is age, ${ }^{21}$ and thus our initial analyses examined the relationship between age and the CSF biomarkers as well as age and the imaging measures (MWF, $R_{1}$, and $R_{2}$ ). To examine the relationships between age and CSF biomarkers, Pearson correlations were calculated in $\mathrm{R}$ (version 3.2.1). ${ }^{22}$ Significant correlations were defined as $P<.05$, Bonferroni-corrected for 28 independent comparisons.

\begin{tabular}{ll}
\hline \multicolumn{2}{l}{ Table. Demographic Characteristics of Participants } \\
\hline Characteristic & Mean (No. of Days) \\
\hline No. of participants & 71 \\
\hline Age, mean (SD), y & $61.6(6.4)$ \\
\hline Sex & 19 \\
\hline Male & 52 \\
\hline Female & $28(2 / 26) / 43$ \\
\hline APOE4 carriers (e4e4/e4e3)/noncarriers & \\
\hline AD parental history & 54 \\
\hline Positive & 17 \\
\hline Negative & $16.96(2.57)$ \\
\hline Education, y & $29.36(1.00)$ \\
\hline MMSE score & \\
\hline RAVLT score & $6.74(1.90)$ \\
\hline Trial 1 & $45.96(6.36)$ \\
\hline Trials 2-5 & $10.97(2.88)$ \\
\hline Delay & $60.07(22.49)$ \\
\hline TMT Part B, s & $37.22(0-205)$ \\
\hline $\begin{array}{l}\text { No. of days between MRI and lumbar puncture, } \\
\text { mean (range), } d\end{array}$ & \\
\hline
\end{tabular}

Abbreviations: AD, Alzheimer disease; APOE4, apolipoprotein E4 allele; MMSE, Mini-Mental State Examination; MRI, magnetic resonance imaging; RAVLT, Rey Auditory Verbal Learning Test; TMT, Trail-Making Test.

Age-related changes in $R_{1}, R_{2}$, and MWF were examined by fitting linear models at each brain voxel contained within a white matter mask (eFigure 2 in the Supplement). Each model examined $R_{1}, R_{2}$ and MWF as a function of age, while accounting for sex, $A P O E$ carrier status, log-transformed normalized $\mathrm{WMH}$ volume, and the Framingham cardiovascular risk score due to known effects on brain microstructure. ${ }^{23}$ We examined education level and family history as additional nuisance variables; however, no significant relationships were observed by including these variables. Statistical maps were thresholded at a level of $P<.05$, corrected for multiple comparisons using the false discovery rate $(\mathrm{FDR})^{24}$ at the voxel level.

\section{Relationships Between CSF Biomarkers and MWF}

Voxelwise linear regressions were fit to examine relationships between $R_{1}, R_{2}$, and MWF and concentration levels of CSF biomarkers. Individual CSF biomarkers, as well as biomarker ratios with $\mathrm{A} \beta 42$, were used as predictor variables, while age, sex, APOE status, Framingham cardiovascular risk score, and log-transformed normalized WMH volume were covariates. Analyses were constrained to white matter and the FDR corrected for multiple comparisons.

After establishing significant findings between age and relaxometry measures as well as between $R_{1}, R_{2}$, and MWF and CSF biomarkers, we used additional models to examine whether concentrations of CSF biomarkers moderated the effect of age on the brain's MWF, $R_{1}$, and $R_{2}$. Predictors in these voxelwise models included age, CSF biomarker concentration, and the interactions of CSF biomarker concentration with age, as well as the covariates of sex, APOE, log-transformed normalized WMH volume, and Framingham cardiovascular risk score. 


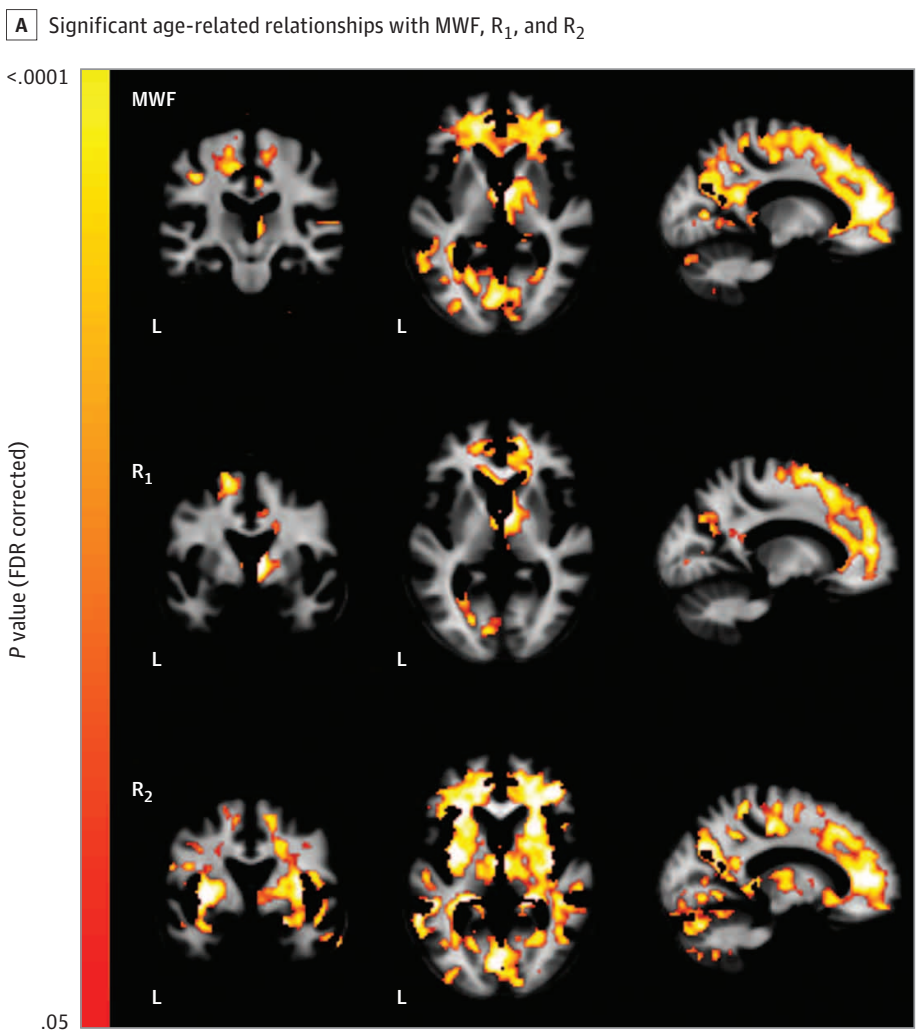

B Mean $M W F, R_{1}$, and $R_{2}$ from significant age decline
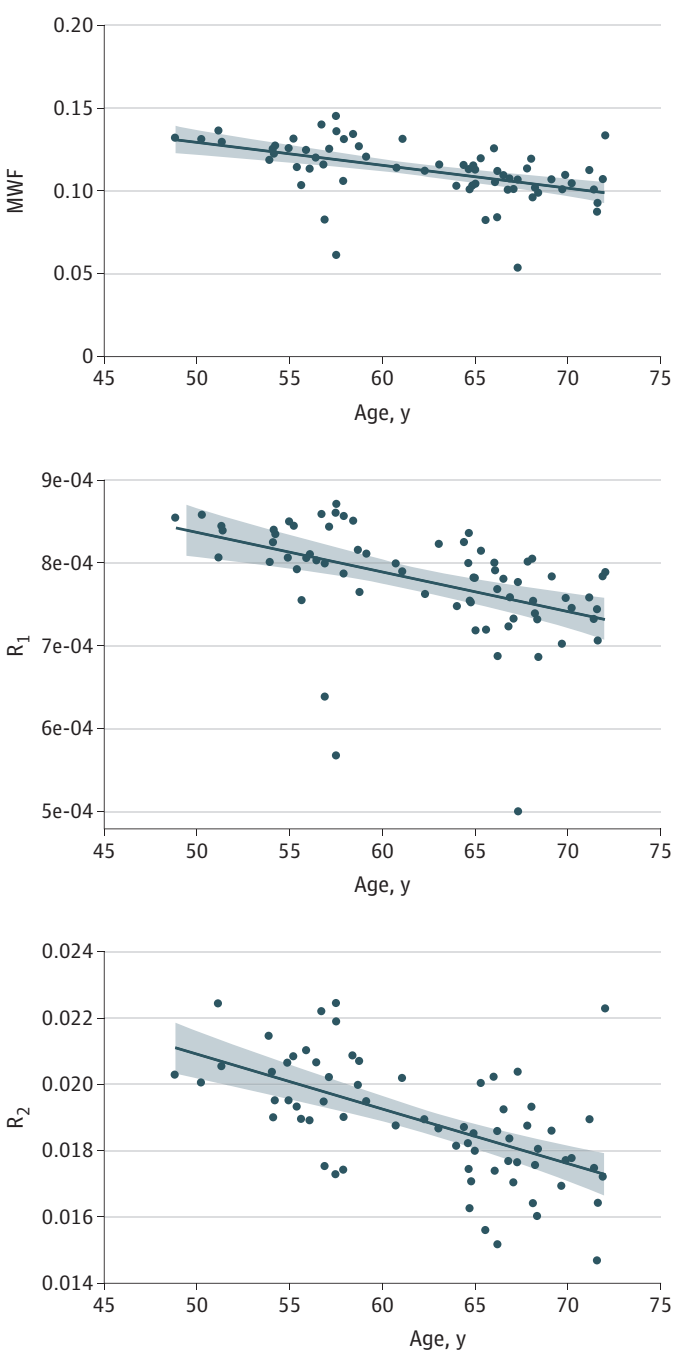

A, Significant $(P<.05$, false discovery rate [FDR] corrected) age-related relationships with myelin water fraction (MWF) (top row), $R_{1}$ (middle row), and $R_{2}$ (bottom row) overlaid on the group mean MWF map. Negative relationships were observed across widespread white matter, with late myelinating areas, including frontal and temporal white matter, having the strongest age-related declines. $B$, representative scatterplot depicts the mean $M W F, R_{1}$, and $R_{2}$ calculated from areas of significant age decline plotted against the age of each participant. Scatter points represent individual mean parameter (MWF, $R_{1}$, and $R_{2}$ ) values and the solid trend line represents the line of best fit with age.

\section{Results}

Changes in CSF Biomarkers and Imaging Measures With Age Age at time of lumbar puncture was not significantly associated with CSF biomarkers (Pearson correlations shown in eTable 1 in the Supplement).

Widespread age-related changes $(P<.05$, FDR corrected) were observed, particularly in late myelinating brain regions such as frontal white matter and the genu of the corpus callosum (Figure 1). Myelin water fraction was negatively related to age, suggesting an overall decrease in myelin content with aging. $\mathrm{R}_{1}$ decreased in similar brain regions, including frontal white matter and the genu of the corpus, while $\mathrm{R}_{2}$ decreased with age across most white matter. Summary information is provided in eTable 2 in the Supplement. These findings suggest alterations to microstructural white matter and perhaps specifically in myelin content; however, such changes may be a result of increases of bulk water content or other biologically based changes. ${ }^{25}$ Such findings are consistent with reported literature of age-related declines of white matter during typical and atypical aging. ${ }^{26}$

\section{Relationships Between CSF Biomarkers}

and Imaging Measures

Voxelwise regressions between CSF biomarkers and imaging measures revealed widespread and robust main effect associations. Lower $A \beta 42$ was associated with decreased MWF, $R_{1}$, 
and $R_{2} \cdot R_{2}$ displayed the most extensive relationships, followed by MWF and $\mathrm{R}_{1}$ (eTables 3-5 in the Supplement). Relationships were primarily located within the left hemispheric angular gyrus white matter for MWF and $\mathrm{R}_{1}$, while associations with $\mathrm{R}_{2}$ were found across the left hemispheric temporal and parietal white matter and inferior longitudinal fasciculus. Other less localized white matter regions were additionally implicated (eFigure 3 in the Supplement).

We observed significant negative relationships $(P<.05$, FDR corrected) between MWF, $\mathrm{R}_{1}$, and $\mathrm{R}_{2}$ and CSF biomarkers of Ttau, Ttau/A $\beta 42$, Ptau181, Ptau181/A $\beta 42$, sAPP $\beta$, and sAPP $\beta / A \beta 42$. Representative findings between $M W F$ and SAPP $\beta / A \beta 42$ are shown in Figure 2 while relationships between $M W F, R_{1}$, and $R_{2}$ and other CSF biomarkers are shown in eFigures 4-6 in the Supplement. We highlight the negative association between MWF and SAPP $\beta / A \beta 42$ in representative scatterplots in Figure 3. Relationships with MWF were extensive across white matter and discovered in the frontal, temporal, parietal, and cerebellar white matter. Soluble amyloid precursor protein/A 342 showed the strongest effect with regions known to be preferentially affected by $\mathrm{AD}$, including temporal and frontal white matter, the body of the corpus callosum, and the cingulum. ${ }^{27}$ We found negative relationships between CSF biomarkers and $\mathrm{R}_{1}$ in the cingulum, inferior fronto-occipital fasciculus, and the superior longitudinal fasciculus, with extensive relationships occurring with sAPP $\beta / A \beta 42$. We also observed negative associations with $R_{2}$, with relations between $R_{2}$ and Ptau181/A 342 having the largest impact.

CSF Biomarkers Moderate Myelin Content Age Relationships We observed significant $(P<.05$, FDR corrected) ageby-biomarker interactions. In particular, we found relationships between MWF and Ptau181/Aß42 in left hemispheric superior frontal gyral white matter and portions of the left superior longitudinal fasciulus in which higher levels of Ptau181/A 442 resulted in an increased MWF decline with age (Figure 4). We provide a summary and depiction of the interaction results in eTable 6 and eFigure 7 in the Supplement.

\section{Discussion}

Alzheimer disease disrupts critical metabolic processes that ultimately lead to neurodegeneration; however, the effect of AD pathology on white matter-especially myelin-is still incompletely characterized. Using CSF biomarkers of AD together with quantitative relaxometry measures in cognitively asymptomatic middle-aged adults enriched for AD risk, we have demonstrated significant associations between $\mathrm{AD}$ pathology and measures of myelin health in vivo. Elevated concentrations of Ttau, Ptau181, and SAPP $\beta$-along with elevated ratios of Ttau/A $\beta 42$, Ptau181/A $\beta 42$, and sAPP $\beta / A \beta 42-$ were robustly associated with decreased $M W F, R_{1}$, and $R_{2}$ across widespread brain regions. The effect of age on the trajectory of MWF was moderated by ratios of Ptau181/A $\beta 42$ and Ttau/A 342 , with elevated ratios leading to accelerated MWF decline. This is the first study, to our knowledge, to demonstrate relationships between $\mathrm{AD}$ pathology and relaxometry measures and
Figure 2. Regional White Matter Myelin Content, as Measured by Myelin Water Fraction (MWF), Associated With Soluble Amyloid Precursor Protein/ $\beta$-Amyloid 42 (SAPP $\beta / A \beta 42)$

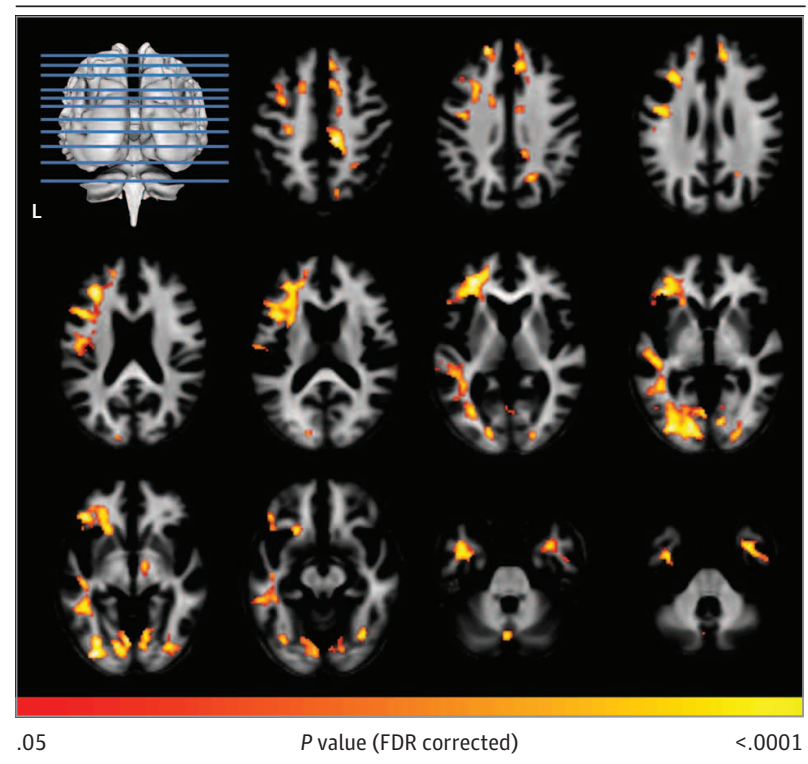

Representative axial slices depicting areas of significant relationship between MWF and SAPP $\beta / A \beta 42$ overlaid on the group mean MWF map. Widespread white matter was observed to be negatively related to levels of SAPP $\beta / A \beta 42$. Results are false discovery rate (FDR) corrected for multiple comparisons $(P<.05)$.

further reinforce recent findings that $\mathrm{AD}$ pathology has an important impact on white matter microstructure. ${ }^{4}$

Our findings provide evidence that risk factors for developing $\mathrm{AD}$ are related to alterations of myelin content. Specifically, we find that age highly associates with $M W F, R_{1}$, and $R_{2}$ decreases. These findings agree with studies showing white matter volume and microstructure to follow a negative gradient throughout later stages of life. ${ }^{13,28}$ Our results agree with hypotheses of late myelinating brain regions ${ }^{29,30}$ being the first to degenerate with age ${ }^{26}$ and implicated in $\mathrm{AD} .{ }^{31}$ Together, this suggests that white matter alterations may be centrally involved in $\mathrm{AD}$ pathogenesis. Analyses of MWF and age were performed using linear regressions as the age range of participants did not appear to capture the nonlinearity of the MWF trajectory. However, the brain follows a nonlinear trajectory, ${ }^{26,32}$ and thus future studies using larger samples and wider age ranges should examine whether MWF follows such a nonlinear trajectory. Such information would improve understanding about the timing of AD pathology, including the initial periods of myelin decline.

We additionally show robust associations between CSF biomarkers and myelin content as measured by MWF, $R_{1}$, and $R_{2}$. Amyloid pathology has classically been linked with processes underlying neuronal degeneration; however, studies have revealed myelin and oligodendrocytes to be especially vulnerable to impairments of $\mathrm{A} \beta$ pathology. ${ }^{5}$ Myelin basic protein, a canonical protein component of myelin, has recently been shown to bind $A \beta$ and inhibit $A \beta$ fibril formation, possibly playing a role in regulating the deposition of $A \beta 42$ and the 

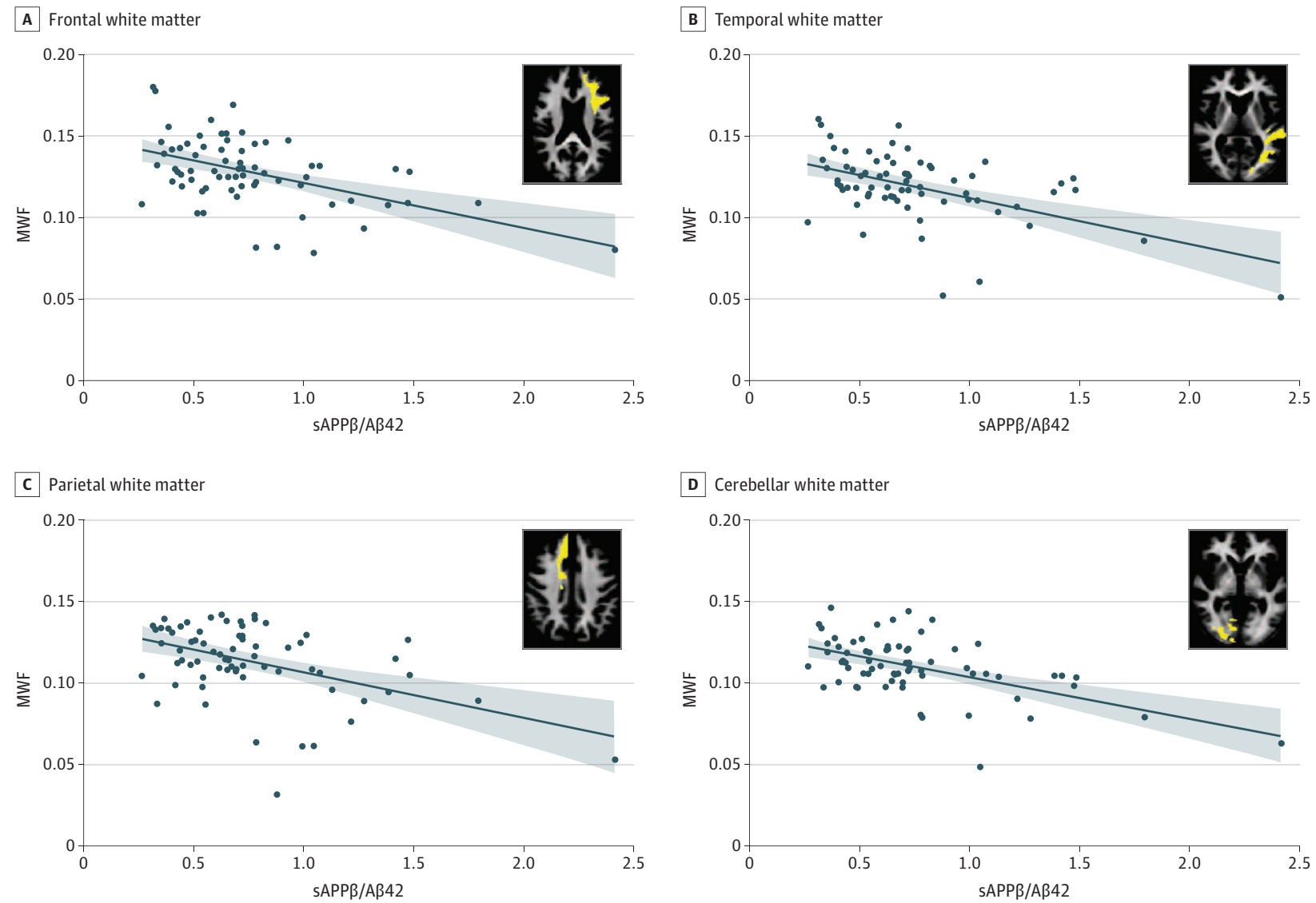

Clusters of 50 voxels or greater were determined from false discovery rate-corrected associations between MWF and sAPP $\beta / A \beta 42$. Mean MWF from the indicated significant clusters were calculated and plotted against SAPP $\beta / A \beta 42$. Each point in the scatter represents mean MWF and corresponding sAPP $\beta / A \beta 42$ from 1 participant $(n=71)$.

formation of amyloid plaques in parenchyma. ${ }^{33,34}$ Therefore, the loss of myelin and decreases in concentrations of myelin basic protein may promote accelerated $\mathrm{A} \beta 42$ depositions and result in increased amyloid plaque formation. Studies have additionally demonstrated that myelin abnormalities occur prior to axon defects from tau proteins inhibiting axon transport, ${ }^{35}$ meaning that myelination defects precede overt amyloid and tau pathologies. ${ }^{36}$

Interestingly, we observed strong associations of decreased myelin content with increased ratios of SAPP $\beta$ and sAPP $\beta / A \beta 42$, with $S A P P \beta / A \beta 42$ having the greatest sensitivity. $\beta$-Amyloid is a product of amyloid precursor protein cleavage by $\beta$-secretase, which ultimately leads to the formation of amyloid plaques. ${ }^{5}$ Animal studies using immunohistochemistry and electron microscopy on the triple-transgenic AD mouse, which harbors the amyloid precursor protein transgene, have found localized alterations in myelination and oligodendrocyte marker expression prior to the manifestation of amyloid and tau pathologies. ${ }^{36}$ Findings from these previous studies in the context of the current study raise questions about the role of SAPP $\beta$ in myelin alterations of AD pathology. The remaining challenge of deciphering the causal mechanisms of these changes is determining if aberrant proteolytic processing of the amyloid precursor protein causes subsequent myelin damage or if degradation of myelin results in altered amyloid precursor protein processing. We need further animal and human studies to elucidate this causal pathway.

Our findings also indicate that the strength of the relationship between MWF and age is altered by the ratios of Ptau181/ $\mathrm{A} \beta 42$ and Ttau/Aß42. While tau pathology may follow amyloid pathology, hyperphosphorylation of tau protein also occurs during the repair of the myelin sheath. ${ }^{5}$ Increased concentrations of Ttau and Ptau181 may reflect initial benefits to remyelination ${ }^{5,13}$ and the effects of moderate myelin breakdown with aging. One hypothesis is that extensive timelimited repair and/or unsuccessful repair could lead to protein aggregation and deposition as neurofibrillary tangles. ${ }^{5}$

\section{Limitations}

This study has several limitations. First, while the sample provides a powerful cohort for investigating relationships of CSF biomarkers and brain microstructure, the enriched risk for $\mathrm{AD}$ (large percentage of family history positive- and APOE $\varepsilon 4$ positive individuals), as well as the low percentage of males 


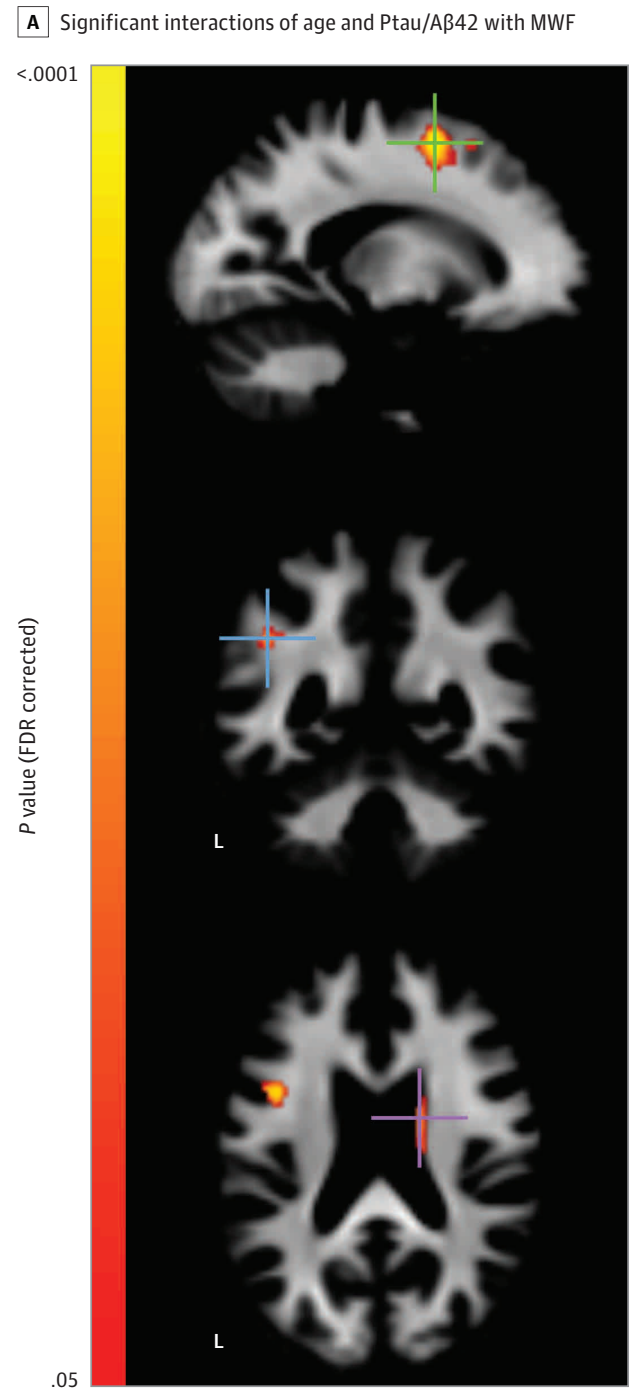

B Changing age relationships with dissimilar Ptau/Aß42 ratio
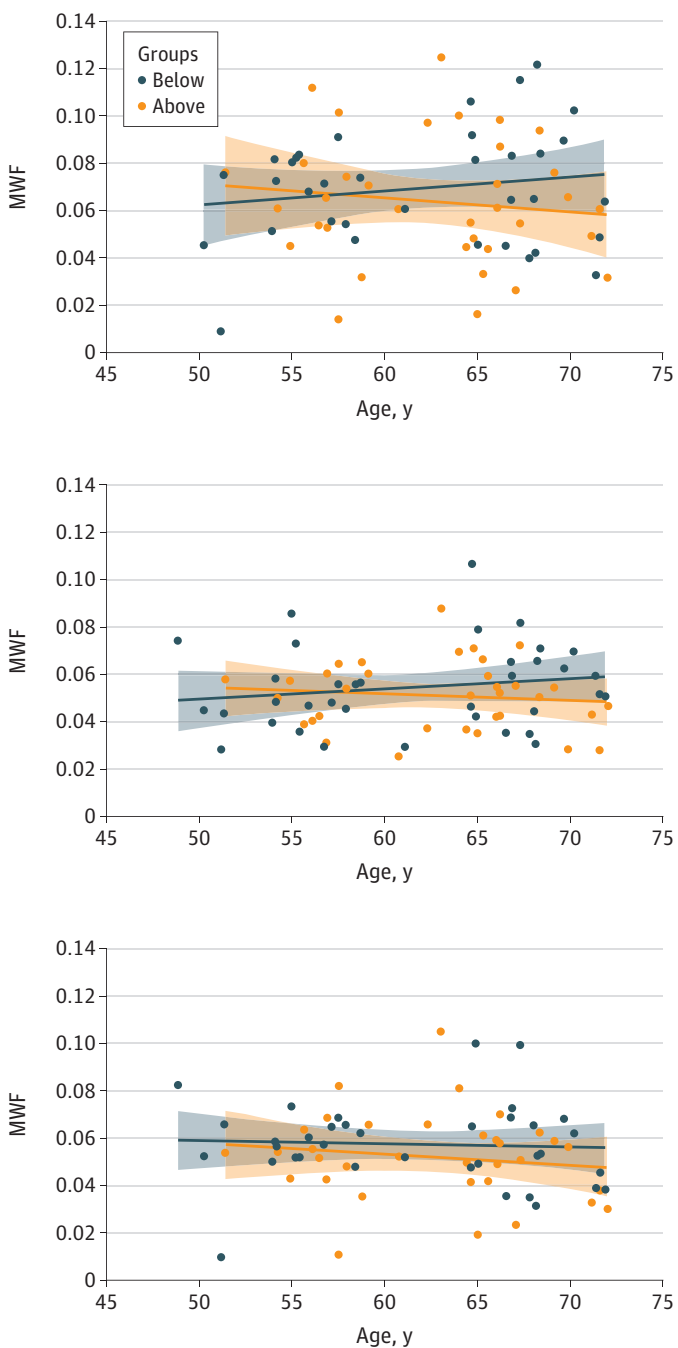

A, Representative illustration of significant interactions of age and Ptau181/Aß42 with MWF. Myelin water fraction from the peak voxel (as indicated by the cross-hair) was extracted and participants were subsequently split into 2 groups by the Ptau181/A 42 ratio median. B, Myelin water fraction was plotted and fit against participant age for each group. These plots demonstrate the changing age relationship with dissimilar Ptau181/A 342 ratio.

(19\%), could limit the generalizability of our findings. Family history was found to have a nonsignificant effect on the findings of the current study. APOE status was included as a nuisance variable; however, it is possible that $A P O E$ allele status may impact brain aging ${ }^{26,28}$ and that future studies examining such relationships could be informative. Second, while qualitative agreement has been observed between mcDESPOT MWF and histology, ${ }^{37}$ and strong evidence has been provided to support the application of mcDESPOT MWF, ${ }^{29,30,38-40}$ future studies investigating the specificity and sensitivity of mcDESPOTderived MWF measurements compared with additional factorssuch as changes to brain volume, water content, iron load, or microstructural lipids and proteins-are necessary. While there was an overlap in findings with $M W F, R_{1}$, and $R_{2}$, associations in different brain structures between brain measures were also observed, suggesting that these measures are complimentary but may elucidate differential microstructural processes. ${ }^{29}$ Last, cross-sectional analyses have permitted us to examine the associations between CSF biomarkers and neuroimaging measures. However, these findings should be explored in larger samples and extended in longitudinal samples to evaluate the time course of these relationships.

\section{Conclusions}

Myelin alterations in AD are suspected but understudied in human populations. Using a quantitative MRI technique sensitive to myelination, we measured CSF biomarkers and myelin content to examine the relationships between $\mathrm{AD}$ pathology. Our findings show, for the first time to our knowledge, that decreased concentrations of $A \beta 42$ and elevated 
concentrations of Ttau, Ptau181, sAPP $\beta$, and their ratios with A $\beta 42$ are closely associated with brain myelin content. Furthermore, we show that the age-related decline in myelin content is influenced by the levels of CSF biomarkers. These findings provide further evidence of white matter involvement, and particularly myelin content, in the pathogenesis of $\mathrm{AD}$ and suggest that such alterations may be one of the earliest characteristics of the disease process.

\section{ARTICLE INFORMATION}

Accepted for Publication: June 30, 2016.

Published Online: November 14, 2016.

doi:10.1001/jamaneurol.2016.3232.

Author Affiliations: Waisman Center, University of Wisconsin-Madison (Dean, Kecskemeti, Alexander); Oxford Centre for Functional Magnetic Resonance Imaging of the Brain, University of Oxford, Oxford, England (Hurley); Alzheimer Disease Research Center, University of Wisconsin School of Medicine and Public Health, Madison (O'Grady, Canda, Davenport-Sis, Carlsson, Asthana, Sager, Johnson, Bendlin); Wisconsin Alzheimer Institute, University of Wisconsin School of Medicine and Public Health, Madison (Davenport-Sis, Asthana, Sager, Johnson); Geriatric Research Education and Clinical Center, William S. Middleton Memorial VA Hospital, Madison, Wisconsin (Carlsson, Asthana, Johnson, Bendlin): Clinical Neurochemistry Laboratory, Department of Psychiatry and Neurochemistry, Institute of Neuroscience and Physiology, The Sahlgrenska Academy at the University of Gothenburg, Mölndal Sweden (Zetterberg, Blennow); Department of Molecular Neuroscience, Institute of Neurology, University College London, London, England (Zetterberg); Department of Medical Physics, University of Wisconsin School of Medicine and Public Health, Madison (Alexander); Department of Psychiatry, University of Wisconsin School of Medicine and Public Health, Madison (Alexander).

Author Contributions: Dr Bendlin had full access to all the data in the study and takes responsibility for the integrity of the data and the accuracy of the data analysis.

Concept and design: Dean, Alexander, Bendlin. Acquisition, analysis, or interpretation of data: Dean, Hurley, Kecskemeti, O'Grady, Canda, Davenport-Sis, Zetterberg, Blennow, Asthana, Sager, Johnson, Alexander, Bendlin.

Drafting of the manuscript: Dean, Bendlin. Critical revision of the manuscript for important intellectual content: Dean, Hurley, Kecskemeti, O'Grady, Canda, Davenport-Sis, Zetterberg, Blennow, Asthana, Sager, Johnson, Alexander Bendlin.

Statistical analysis: Dean, Hurley, O'Grady, Canda, Johnson.

Obtaining funding: Blennow, Sager, Johnson, Alexander, Bendlin.

Administrative, technical, or material support: Dean Kecskemeti, O'Grady, Canda, Davenport-Sis, Zetterberg, Blennow, Asthana, Johnson, Alexander. Study supervision: Alexander, Bendlin.

Conflict of Interest Disclosures: Dr Carlsson serves as a site principal investigator for a study that is jointly funded by the National Institutes of Health and Eli Lilly and Company and receives funding from the US Department of Veterans Affairs through the Veterans Affairs Merit grant program. Dr Blennow has served as a consultant at and on advisory boards for IBL International, Fujirebio Europe, Eli Lilly, and Novartis. No other disclosures were reported.
Funding/Support: This study was supported by the Eunice Kennedy Shriver National Institute of Child Health and Human Development (grants R21HD078119 [Dr Alexander], T32 HD007489 [Dr Dean], and P3O HD003352 [Waisman Center]); the National Institute on Aging (grants R01AG027161 [Dr Johnson], R01AG037639 [Dr Bendlin], and ADRC P50AG033514 [Dr Asthana]); the University of Wisconsin Institute for Clinical and Translational Research, funded through a National Center for Research Resources/National Institutes of Health Clinical and Translational Science Award (grant 1UL1RR025011); and a program of the National Center for Research Resources, US National Institutes of Health. The study was also facilitated by the facilities and resources at the Geriatric Research, Education, and Clinical Center of the William S. Middleton Memorial Veterans Hospital, Madison, Wisconsin. Biomarker analyses in this study were supported by grants from the Swedish Research Council, the Torsten Söderberg Foundation at the Royal Swedish Academy of Sciences (Dr Blennow), and the Swedish Alzheimer Foundation (Dr Blennow)

Role of the Funder/Sponsor: The funding sources had no role in the design and conduct of the study; collection, management, analysis, or interpretation of the data; preparation, review, or approval of the manuscript; and decision to submit the manuscript for publication.

Disclaimer: The content is solely the responsibility of the authors and does not necessarily represent the official views of the National Institutes of Health.

Additional Contributions: We thank Jitka Sojkova, MD (Department of Neurology, University of Wisconsin-Madison), for many useful conversations that precipitated this work. Dr Sojkova was not compensated for her contributions.

\section{REFERENCES}

1. Hardy J, Selkoe DJ. The amyloid hypothesis of Alzheimer's disease: progress and problems on the road to therapeutics. Science. 2002;297(5580): 353-356.

2. Bartzokis G, Lu PH, Mintz J. Human brain myelination and amyloid beta deposition in Alzheimer's disease. Alzheimers Dement. 2007;3 (2):122-125.

3. Haight TJ, Landau SM, Carmichael O, Schwarz C, DeCarli C, Jagust WJ; Alzheimer's Disease Neuroimaging Initiative. Dissociable effects of Alzheimer disease and white matter hyperintensities on brain metabolism. JAMA Neurol. 2013;70(8):1039-1045.

4. Bendlin BB, Carlsson CM, Johnson SC, et al. CSF $\mathrm{t}$-tau/Aß42 predicts white matter microstructure in healthy adults at risk for Alzheimer's disease. PLoS One. 2012;7(6):e37720.

5. Bartzokis G. Alzheimer's disease as homeostatic responses to age-related myelin breakdown. Neurobiol Aging. 2011;32(8):1341-1371.
6. Roher AE, Weiss N, Kokjohn TA, et al. Increased $A \beta$ peptides and reduced cholesterol and myelin proteins characterize white matter degeneration in Alzheimer's disease. Biochemistry. 2002;41(37): 11080-11090.

7. Zetterberg H, Wahlund L-O, Blennow K. Cerebrospinal fluid markers for prediction of Alzheimer's disease. Neurosci Lett. 2003;352(1):6769.

8. Blennow K, Hampel H. CSF markers for incipient Alzheimer's disease. Lancet Neurol. 2003;2(10): 605-613.

9. Flood DG, Marek GJ, Williams M. Developing predictive CSF biomarkers-a challenge critical to success in Alzheimer's disease and neuropsychiatric translational medicine. Biochem Pharmacol. 2011;81 (12):1422-1434.

10. Hampel H, Teipel SJ, Fuchsberger T, et al. Value of CSF beta-amyloid1-42 and tau as predictors of Alzheimer's disease in patients with mild cognitive impairment. Mol Psychiatry. 2004;9(7):705-710.

11. Glodzik L, de Santi S, Tsui WH, et al. Phosphorylated tau 231, memory decline and medial temporal atrophy in normal elders. Neurobiol Aging. 2011;32(12):2131-2141.

12. Jones DK, Knösche TR, Turner R. White matter integrity, fiber count, and other fallacies: the do's and dont's of diffusion MRI. Neuroimage. 2013;73: 239-254.

13. Bartzokis G. Age-related myelin breakdown: a developmental model of cognitive decline and Alzheimer's disease. Neurobiol Aging. 2004;25(1):5 18.

14. MacKay A, Whittall K, Adler J, Li D, Paty D, Graeb D. In vivo visualization of myelin water in brain by magnetic resonance. Magn Reson Med. 1994;31(6):673-677.

15. Deoni SCL, Rutt BK, Arun T, Pierpaoli C, Jones DK. Gleaning multicomponent $\mathrm{T} 1$ and $\mathrm{T} 2$ information from steady-state imaging data. Magn Reson Med. 2008;60(6):1372-1387.

16. Blennow K, Hampel H, Weiner M, Zetterberg $\mathrm{H}$. Cerebrospinal fluid and plasma biomarkers in Alzheimer's disease. Nat Rev Neurol. 2010;6(3):131144.

17. Sager MA, Hermann B, La Rue A. Middle-aged children of persons with Alzheimer's disease: APOE genotypes and cognitive function in the Wisconsin Registry for Alzheimer Prevention. J Geriatr Psychiatry Neurol. 2005;18(4):245-249.

18. Deoni SCL, Matthews L, Kolind SH. One component? two components? three? the effect of including a nonexchanging "free" water component in multicomponent driven equilibrium single pulse observation of T1 and T2. Magn Reson Med. 2013; 70(1):147-154

19. Folstein MF, Folstein SE, McHugh PR. "Mini-mental state": a practical method for grading the cognitive state of patients for the clinician. J Psychiatr Res. 1975;12(3):189-198.

20. Palmqvist $\mathrm{S}$, Zetterberg $\mathrm{H}$, Blennow $\mathrm{K}$, et al. Accuracy of brain amyloid detection in clinical 
practice using cerebrospinal fluid $\beta$-amyloid 42: a cross-validation study against amyloid positron emission tomography. JAMA Neurol. 2014;71(10): 1282-1289.

21. Reitz C, Brayne C, Mayeux R. Epidemiology of Alzheimer disease. Nat Rev Neurol. 2011;7(3):137-152.

22. R Core Team. R: a language and environment for statistical computing. Vienna, Austria: R Foundation for Statistical Computing; 2014. http: //www.R-project.org. Accessed April 20, 2016

23. Bendlin BB, Fitzgerald ME, Ries ML, et al. White matter in aging and cognition: a cross-sectional study of microstructure in adults aged eighteen to eighty-three. Dev Neuropsychol. 2010;35(3):257-277.

24. Benjamini Y, Hochberg Y. Controlling the false discovery rate: a practical and powerful approach to multiple testing. J R Stat Soc Ser B. 1995;57(1):289300. https://www.jstor.org/stable/2346101. Accessed October 16, 2015

25. MacKay A, Laule C, Vavasour I, Bjarnason T, Kolind S, Mädler B. Insights into brain microstructure from the T2 distribution. Magn Reson Imaging. 2006;24(4):515-525.

26. Bartzokis G. Quadratic trajectories of brain myelin content: unifying construct for neuropsychiatric disorders. Neurobiol Aging. 2004; 25(1):49-62.

27. Madsen SK, Ho AJ, Hua X, et al; Alzheimer's Disease Neuroimaging Initiative. 3D maps localize caudate nucleus atrophy in 400 Alzheimer's disease, mild cognitive impairment, and healthy elderly subjects. Neurobiol Aging. 2010;31(8):1312 1325.
28. Bartzokis G, Lu PH, Mintz J. Quantifying age-related myelin breakdown with MRI: novel therapeutic targets for preventing cognitive decline and Alzheimer's disease. J Alzheimers Dis. 2004;6 (6)(suppl):53-59.

29. Deoni SCL, Dean DC III, O'Muircheartaigh J, Dirks H, Jerskey BA. Investigating white matter development in infancy and early childhood using myelin water faction and relaxation time mapping. Neuroimage. 2012;63(3):1038-1053.

30. Dean DC III, O'Muircheartaigh J, Dirks H, et al. Characterizing longitudinal white matter development during early childhood. Brain Struct Funct. 2015;220(4):1921-1933.

31. Braak H, Del Tredici K. The preclinical phase of the pathological process underlying sporadic Alzheimer's disease. Brain. 2015;138(pt 10):28142833.

32. Giedd JN, Blumenthal J, Jeffries NO, et al. Brain development during childhood and adolescence: a longitudinal MRI study. Nat Neurosci. 1999;2(10): 861-863.

33. Liao M-C, Ahmed M, Smith SO, Van Nostrand WE. Degradation of amyloid beta protein by purified myelin basic protein. J Biol Chem. 2009; 284(42):28917-28925

34. Hoos MD, Ahmed M, Smith SO, Van Nostrand WE. Myelin basic protein binds to and inhibits the fibrillar assembly of A 342 in vitro. Biochemistry. 2009;48(22):4720-4727.
35. Mandelkow EM, Stamer K, Vogel R, Thies E, Mandelkow E. Clogging of axons by tau, inhibition of axonal traffic and starvation of synapses. Neurobiol Aging. 2003;24(8):1079-1085.

36. Desai MK, Sudol KL, Janelsins MC, Mastrangelo MA, Frazer ME, Bowers WJ. Triple-transgenic Alzheimer's disease mice exhibit region-specific abnormalities in brain myelination patterns prior to appearance of amyloid and tau pathology. Glia. 2009;57(1):54-65.

37. Hurley SA, Mossahebi PM, Samsonov AA. Multicomponent relaxometry (mcDESPOT) in the shaking pup model of dysmyelination. Paper presented at: International Society for Magnetic Resonance in Medicine 2010 Annual Meeting; May 6, 2010; Stockholm, Sweden. http://cds.ismrm .org/protected/10MProceedings/files/4516_88.pdf. Accessed March 15, 2016.

38. Kitzler HH, Su J, Zeineh M, et al. Deficient MWF mapping in multiple sclerosis using 3D whole-brain multi-component relaxation MRI. Neuroimage. 2012;59(3):2670-2677.

39. Kolind $\mathrm{S}$, Matthews $\mathrm{L}$, Johansen-Berg $\mathrm{H}$, et al. Myelin water imaging reflects clinical variability in multiple sclerosis. Neuroimage. 2012;60(1):263-270.

40. Spader HS, Ellermeier A, O'Muircheartaigh J, et al. Advances in myelin imaging with potential clinical application to pediatric imaging. Neurosurg Focus. 2013;34(4):E9. 